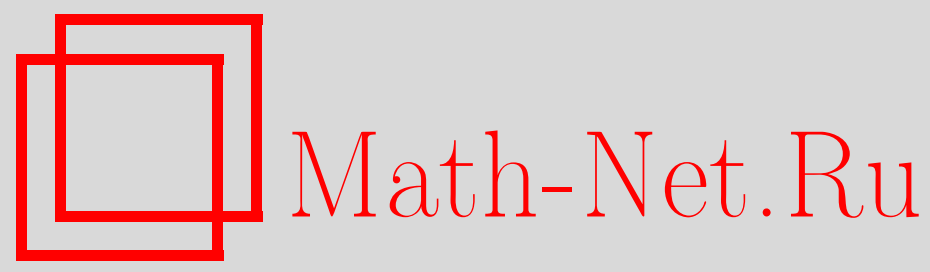

С. А. Шаповалов, Инвариант глубины для групповых действий на счетных фазовых пространствах, Матем. заметки, 1999, том 65, выпуск 6, 893-907

DOI: https://doi.org/10.4213/mzm1125

Использование Общероссийского математического портала Math-Net.Ru подразумевает, что вы прочитали и согласны с пользовательским соглашением http://www . mathnet.ru/rus/agreement

Параметры загрузки:

IP : 54.81 .137 .203

26 апреля 2023 г., 15:54:16

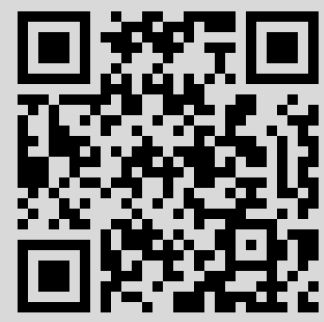




\section{ИНВАРИАНТ ГЛУБИНЫ ДЛЯ ГРУППОВЫХ ДЕЙСТВИЙ НА СЧЕТНЫХ ФАЗОВЫХ ПРОСТРАНСТВАХ}

\section{С. А. Шаповалов}

Исследованная ранее автором для случая действия группы $\mathbb{Z}$ конструкция глубины счетной символической системы обобщается на случай действия произвольной конечнопорожденной абелевой группы. Изучается новое свойство, названное однородностью глубины. Глубина является топологическим инвариантом счетных символических систем, принимающим значение в множестве не более чем счетных ординальных чисел. В работе описываются множество возможных значений инварианта глубины и метод построения динамических систем с произвольньм допустимьм значением глубины.

Библиография: 6 названий.

В этой статье обобщается исследованная автором в работе [1] конструкция топологического инварианта глубины динамической системы на случай действия счетных конечнопорожденных абелевых групп. Приводится доказательство теоремы о множестве ординалов, которые могут быть значениями глубины счетных символических систем.

Идея указанного инварианта навеяна конструкцией центра динамической системы, приведенной Биркгофом в [2], а также результатом А. Г. Майера, построившего в [3] примеры динамических систем в $\mathbb{R}^{3}$ с произвольным счетным ординалом в качестве глубины центра (изложение результатов Майера см. также в книге В. В. Немьщкого и В. В. Степанова [4]).

Существуют различные модификации понятия глубины центра. Описание их конструкции и связи между ними можно найти, например, в работе Д. Неймана [5]. Тем не менее, введенньй в [1] и изучаемьй в настоящей статье инвариант глубины является новым. (Во избежание недоразумений, связанных с терминологией, заметим, что если $C$ - центр некоторой динамической системы, то ее глубина центра и глубина $C$, вообще говоря, разные понятия.)

В работе [6] Д. В. Аносов высказал замечание о том, что при изучении глубины центра (и родственных инвариантов) решающую роль играет рассмотрение счетных замкнутых инвариантных подмножеств бернуллиевского топологического каскада. Исходя из этого соображения, мы будем рассматривать лишь символические динамические системы со счетными замкнутыми инвариантньми фазовыми пространствами.

1. Определения, обозначения, основные понятия. Пусть $G$ - конечнопорожденная абелева группа бесконечного порядка и $A$ - конечньй алфавит; для простоты будем считать, что $A=\{0,1\}$. Определим на $A$ операцию сложения:

$$
0+0=0, \quad 0+1=1, \quad 1+0=1, \quad 1+1=0 .
$$


Рассмотрим $\Omega=\Omega(G, A)=\{x \mid x: G \rightarrow A\}$ - пространство всех отображений из $G$ в $A$. Элементы $x \in \Omega$ будем назьвать конфигурациями на $G$ со значениями в $A$. Фазовыми пространствами всех рассматриваемых нами в этой работе символических систем будут служить счетные подмножества в $\Omega$.

Введем на пространстве $\Omega$ тихоновскую топологию покоординатной сходимости: $x_{n} \rightarrow x_{0}$ при $n \rightarrow \infty, x_{n} \in \Omega, x_{0} \in \Omega$, если для каждого конечного подмножества $G_{0} \subset G$ найдется число $N$ такое, что при всех $n>N$ и всех $g \in G_{0}$ вьполнено равенство $x_{n}(g)=x_{0}(g)$. Указанная топология метризуема; кроме того, $\Omega$ является метрическим компактом.

Зафиксируем некоторую систему образующих $C$ группы $G$. Назовем графом Кәли zр уnпь $G$, соответствуюшим системе $C$, неориентированньй граф $\Gamma=\Gamma(G, C)$, вершинами которого служат элементы группы $G$, причем вершины $g$ и $h$ соединены ребром тогда и только тогда, когда найдется элемент $c \in C$ такой, что $g=c h$ или $g=c^{-1} h$.

Введем следующие обозначения: $d: G \rightarrow \mathbb{Z}^{+}{ }_{-}$целочисленная функция на $G$, значение которой $d(g)$ равно длине кратчайшего пути, состоящего из ребер графа $Г$ и соединяющего $е$ и $g$ как вершины этого графа $(e-$ единица групшы $G) ; p: G \times G \rightarrow \mathbb{Z}^{+}$- метрика на $G$, порожденная функцией $d$, т.е. $p(g, h)=d\left(g h^{-1}\right)$ - длина кратчайшего пути, соединяюшего $g$ и $h$ как вершины графа $\Gamma$.

В дальнейшем нам потребуется определение операции сложения для конфигураций: $(x+y)(g)=x(g)+y(g)$.

Рассмотрим действие $T$ групшы $G$ на пространстве $\Omega$, задаваемое формулой

$$
\left(T_{g} x\right)(h)=x\left(g^{-1} h\right)
$$

Нас интересуют символические динамические системы $(M, T)$, полученные ограничением действия $T$ на непустые не более чем счетные замкнутые $T$-инвариантные подмножества $M$ пространства $\Omega$. Обозначим через $\Sigma=\Sigma(G, A) \subset \Omega(G, A)$ класс фазовых пространств $M$ всех динамических систем $(M, T)$ описанного вида.

Две динамические системы $\left(M_{1}, T\right)$ и $\left(M_{2}, T\right)$ с фазовьми пространствами из $\Sigma$ называются (топологически) сопряженными, если найдется гомеоморфизм $h: M_{1} \rightarrow M_{2}$, коммутирующий с действием $T$, т.е. такой, что при всех $g \in G$ вьполнено $h T_{g}=T_{g} h$.

В следующем пунктемы строим отображение из класса $\Sigma(G, A)$ в множество не более чем счетных ординалов, инвариантное относительно топологической сопряженности. Это отображение назовем глубиной. Мы докажем, что всякое непредельное (и только непредельное) ординальное число может быть глубиной некоторой динамической системы. Приводится явное построение, при помощи которого можно предъявить примеры динамических систем с любой возможной глубиной.

2. Глубина относительно взятия множества предельных точек. Пусть $M-$ некоторое непустое не более чем счетное замкнутое множество в топологическом пространстве $(X, \tau)$. Обозначим через $M^{\prime}$ множество его предельных точек. Полученное множество $M^{\prime}$ замкнуто. Рассмотрев совокупность всех предельных точек в $M^{\prime}$, получим множество $M^{\prime \prime}$. Последовательно применяя процедуру удаления изолированных точек, индуктивно определим множества

$$
M^{(0)}:=M, \quad M^{(1)}:=M^{\prime}, \quad M^{(2)}:=M^{(1)^{\prime}}, \quad \ldots, \quad M^{(n)}:=M^{(n-1)^{\prime}} .
$$


Продолжим этот процесс на счетные трансфинитные числа. Пересечение множеств $M^{(n)}$ по всем конечным $n$ непусто (как пересечение счетной системы убывающих компактов). Положим

$$
M^{(\omega)}:=\left(\bigcap_{n=0}^{\infty} M^{(n)}\right)^{\prime}
$$

В общем случае для не более чем счетного ординала $\alpha$ определение $\alpha$-го производного множества выглядит следующим образом:

$$
M^{(\alpha)}:=\left(\bigcap_{\beta<\alpha} M^{(\beta)}\right)^{\prime} .
$$

В результате получаем цепочку убывающих не более чем счетных множеств:

$$
M^{(0)} \supset M^{(1)} \supset \cdots \supset M^{(\omega)} \supset \cdots \supset M^{(\alpha)} \supset M^{(\alpha+1)} \supset \cdots,
$$

причем для непустых $M^{(\alpha)}$ включения $M^{(\alpha)} \supset M^{(\alpha+1)}$ строгие, так как в этом случае $M^{(\alpha)}$ имеет изолированные точки. Согласно теореме Кантора о несчетности множества всех не более чем счетных ординалов процесс оборвется на некотором шаге $\alpha$ не вьше счетного трансфинитного числа, т.е. $M^{(\alpha)}$ окажется пустым.

ОПредЕлЕниЕ. Минимальное ординальное число $\alpha$ со свойством $M^{(\alpha)}=\varnothing$ называется глубиной множсества $M \subset X$ (относительно топологии $\tau$ на пространстве $X$ ) и обозначается через $\operatorname{depth}(M)$ :

$$
\operatorname{depth}(M):=\min \left\{\alpha \mid M^{(\alpha)}=\varnothing\right\}
$$

ОПРЕДЕЛЕНИЕ. Глубиной динамической системы $(M, T)$, где $M$-некотороене более чем счетное замкнутое $T$-инвариантное подмножество $\Omega(G, A)$, называется глубина ее фазового пространства $M$ относительно тихоновской топологии на $\Omega$.

Заметим, что операция взятия производного множества переводит $\Sigma$ в $\Sigma$. Действительно, замкнутость и $T$-инвариантность фазового пространства сохраняются при переходе к множеству его предельньх точек.

В работе [4] рассматривались символические динамические системы, фазовыми пространствами которых служат счетные замкнутые инвариантные относительно сдвига множества последовательностей из 0 и 1 . Было доказано, что произвольное непредельное не более чем счетное ординальное число может быть реализовано как глубина некоторой системы такого вида. Основываясь на этом результате, можно без труда построить примеры систем $(M, T)$, фазовые пространства которых $M$ лежат в классе $\Sigma(G, A)$ с произвольньми не более чем счетными глубинами (за исключением предельных ординалов). Действительно, сопоставим каждой счетной символической системе из $\Sigma(\mathbb{Z}, A)$ систему той же глубины из $\Sigma(G, A)$. В наиболее наглядном случае $G=\mathbb{Z}^{2}$ один из 
возможных способов трансформации последовательностей элементов из $A$ в “многомерные" конфигурации приведен в следующей таблице:

$\begin{array}{llcccccccccc}\ldots & 0 & 1 & 0 & 1 & 1 & 1 & 0 & 0 & 1 & \ldots & \\ & & & & & \downarrow & & & & & & \\ & \vdots & \vdots & \vdots & \vdots & \vdots & \vdots & \vdots & \vdots & \vdots & \\ & 0 & 1 & 0 & 1 & 1 & 1 & 0 & 0 & 1 & \ldots & \\ \ldots & 0 & 1 & & & \\ \ldots & 0 & 1 & 0 & 1 & 1 & 1 & 0 & 0 & 1 & \ldots \\ \ldots & 0 & 1 & 0 & 1 & 1 & 1 & 0 & 0 & 1 & \ldots \\ & \vdots & \vdots & \vdots & \vdots & \vdots & \vdots & \vdots & \vdots & \vdots & \\ & & & & & & & & & & \end{array}$

В общем случае достаточно зафиксировать некоторую систему образующих $C$ группы $G$, выбрать образуюший элемент $c \in C$ бесконечного порядка и перевести "одномерные" системы в "многомерные" при помощи отображения $\theta_{c}: \Omega(\mathbb{Z}, A) \rightarrow \Omega(G, A)$, задаваемого формулой $\theta_{c}(x)=y$, где $y(g)=x_{i}$, если $c$ входит $i$ раз в разложение $g$ на образующие. Очевидно, что отображения $\theta_{c}$ сохраняют глубины множеств из $\Sigma(\mathbb{Z}, A)$.

Однако во всех полученных подобньм образом системах глубина "неоднородно распределена" по разным направлениям. Точнее говоря, при ограничении $M$ на различные бесконечные циклические подгрупшы групшы $G$ получающиеся подсистемы обладают разной глубиной. Нас же интересуют лиш системы, у которых совпадают глубины “по всем направлениям". Сформулируем указанное свойство.

ОПРЕДЕЛЕНИЕ. Будем говорить, что динамическая система $(M, T)$ с фазовым пространством $M \in \Sigma(G, A)$ обладает свойством однородности глубины, если для каждого бесконечного подмножества $G_{0}$ групшы $G$ глубина множества $\left\{\left.x\right|_{G_{0}} \mid x \in M\right\}$ (снабженного тихоновской топологией) совпадает с глубиной системы $(M, T)$.

Обозначим через $\widetilde{\Sigma}(G, A)$ класс тех фазовых пространств $M$ из $\Sigma(G, A)$, для которых глубина динамических систем $(M, T)$ однородна.

В дальнейшем мы будем иметь дело только с динамическими системами, глубина которых однородна.

Рассмотрим множество всех не более чем счетных ординалов, которые могут быть глубинами динамических систем с таким свойством. Его структуру полностью описывает следующая теорема.

ТЕОремА. 1) Для каждой бесконечной конечнопорожденной абелевой группь $G$ и каждого непредельного не более чем счетного ординала $\alpha$ найдется замкнутое не более чем счетное $T$-инвариантное множество $M \in \widetilde{\Sigma}(G, A)$, глубина которого однородна и равна $\alpha$.

2) Замкнутых T-инвариантных счетных множеств $M \in \Sigma(G, A)$, глубина которых была бы предельным трансфинитным числом, не существует.

ДокАЗАТЕЛЬСтво. Зафиксируем некоторую систему образуюших $C=\left\{c_{1}, \ldots, c_{r}\right\}$ группы $G$.

1) Рассмотрим класс $\widehat{\Sigma}=\widehat{\Sigma}(G, A) \subset \widetilde{\Sigma}(G, A)$ фазовых пространств $M \subset \Omega(G, A)$, удовлетворяющих следующим условиям:

а) $M$ - не более чем счетное замкнутое $T$-инвариантное подмножество пространства $\Omega(G, A)$; 
б) система $(M, T)$ обладает свойством однородности глубины;

в) все конфигурации из $M$ имеют конечные носители (под носителем мы понимаем множество $\operatorname{supp}(x):=\{g \in G \mid x(g)=1\}) ;$

г) если конфигурация $x$ принадлежит $M$ и $\operatorname{supp}(x)=\left\{g_{1}, \ldots, g_{n}\right\}$, то для любого $i=1, \ldots, n$ конфигурация $x^{\prime}$, у которой $\operatorname{supp}\left(x^{\prime}\right)=\left\{g_{1}, \ldots, g_{i-1}, g_{i+1}, \ldots, g_{n}\right\}$, принадлежит $M^{\prime}$.

Докажем, что каждый непредельньй счетньй ординал может быть реализован как глубина некоторой динамической системы $(M, T)$ с фазовьм пространством $M$ из $\widehat{\Sigma}(G, A)$. Тем самым, будет обосновано утверждение 1$)$ теоремы.

Рассуждения будем вести трансфинитной индукцией по глубине конструируемого множества.

База индукции. В качестве динамической системы, глубина которой равна 1, можно взять систему с фазовым пространством $M_{1}=\{\mathbf{0}\}$, где для всех $g \in G \mathbf{0}(g)=0$.

Лемма 1. Множество $M_{1}$ лежит в классе $\widehat{\Sigma}(G, A)$, и его глубина $\operatorname{depth}\left(M_{1}\right)=1$.

ДокАЗАТЕЛьСтво. Первое утверждение очевидно. Поскольку множество $M_{1}$ одноточечное $\operatorname{depth}\left(M_{1}\right)=1$. Лемма доказана.

Вспомогательная конструкция. Построим множество $M_{2}$, которое нам необходимо в дальнейшем. Положим $M_{2}:=\{x \in \Omega \mid \# \operatorname{supp}(x) \leqslant 1\}$.

Лемма 2. Множество $M_{2}$ лежит в классе $\widehat{\Sigma}(G, A)$, и его глубина $\operatorname{depth}\left(M_{2}\right)=2$.

ДокАЗАТЕЛЬСтво. Изолированными точками в $M_{2}$ являются конфигурации, обращаюшиеся в 1 на некотором элементе группы $G$. Единственная предельная точка в $M_{2}-$ это точка 0. Отсюда следует вьполнение для $M_{2}$ свойств а)-г) из определения класса $\widehat{\Sigma}$, а также то, что

$$
M_{2}^{\prime}=\{\mathbf{0}\}=M_{1}, \quad M_{2}^{\prime \prime}=\varnothing, \quad \operatorname{depth}\left(M_{2}\right)=2 .
$$

Лемма доказана.

Шаг индукции. Пусть $\alpha$ - непредельное счетное трансфинитное число и уже построены динамические системы $\left(M_{\beta}, T\right)$ со всеми непредельными глубинами $\beta<\alpha$, причем фазовыепространства всех этих систем лежат в классе $\widehat{\Sigma}$. Построим множество $M_{\alpha} \in \widehat{\Sigma}$ с глубиной $\alpha$. Так как $\alpha$ непредельно, то корректно определено ординальное число $\alpha-1$.

Возникают две ситуации:

i) $\alpha-1$ - непредельное трансфинитное число;

ii) $\alpha-1$ - предельньй ординал.

i) Пусть $\alpha-1$ - непредельное трансфинитное число. По предположению индукции найдется множество $M_{\alpha-1} \in \widehat{\Sigma}$ такое, что $\operatorname{depth}\left(M_{\alpha-1}\right)=\alpha-1$. Определим

$$
M_{\alpha}:=M_{\alpha-1}+M_{2}
$$

(здесь и далее под суммой двух множеств понимается множество попарных сумм их элементов). Докажем, что $M_{\alpha} \in \widehat{\Sigma}$ и $\operatorname{depth}\left(M_{\alpha}\right)=\alpha$. 
ЛЕмма 3. При всех $\beta<\alpha-1$

$$
M_{\alpha}^{(\beta)}=M_{\alpha-1}^{(\beta)}+M_{2}
$$

ДокАЗАтЕльСтво. Рассуждения будем вести трансфинитной индукцией по $\beta$. При $\beta=0$ утверждение верно в силу определения множества $M_{\alpha}$.

Пусть доказываемое равенство выполнено при всех $\beta<\gamma$ для некоторого $\gamma<\alpha-1$. Покажем, что

$$
M_{\alpha}^{(\gamma)}=M_{\alpha-1}^{(\gamma)}+M_{2}
$$

Положим

$$
E=\bigcap_{\beta<\gamma} M_{\alpha}^{(\beta)}, \quad F=\bigcap_{\beta<\gamma} M_{\alpha-1}^{(\beta)} .
$$

Из предположения индукции следует, что $E=F+M_{2}$. Действительно, если $x \in F+M_{2}$, то найдутся $y \in F$ и $z \in M_{2}$ такие, что $x=y+z$. По построению множества $F$ при всех $\beta<\gamma$ имеем $y \in M_{\alpha-1}^{(\beta)}$, поэтому $x=y+z \in M_{\alpha-1}^{(\beta)}+M_{2}=M_{\alpha}^{(\beta)}$. Следовательно,

$$
x \in \bigcap_{\beta<\gamma} M_{\alpha}^{(\beta)}=E .
$$

Таким образом, $E \supset F+M_{2}$.

Докажем обратное вложение. Пусть $x \in E$. Тогда для любого $\beta<\gamma$ найдутся $y_{\beta} \in M_{\alpha-1}^{(\beta)}$ и $z_{\beta} \in M_{2}$ такие, что $x=y_{\beta}+z_{\beta}$. Поэтому при $\beta<\gamma$ непусты множества $X(\beta):=\left\{(y, z) \in M_{\alpha-1}^{(\beta)} \times M_{2} \mid y+z=x\right\}$. Множества $X(\beta)$ замкнуты (в топологии прямого произведения) и убывают с ростом $\beta$. Поэтому $\bigcap_{\beta<\gamma} X(\beta) \neq \varnothing$. Пусть $(y, z) \in$ $\bigcap_{\beta<\gamma} X(\beta)$. Тогда $x=y+z \in \bigcap_{\beta<\gamma} M_{\alpha-1}^{(\beta)}+M_{2}=F+M_{2}$, что и требовалось доказать. Для доказательства леммы требуется установить, что $E^{\prime}=F^{\prime}+M_{2}$. Так как $F^{\prime}=$ $M_{\alpha-1}^{(\gamma)}$, а $\gamma<\alpha-1$, множество $F^{\prime}$ непусто, а поскольку $E \supset F$, и $E^{\prime} \neq \varnothing$.

Покажем, что $E^{\prime} \supset F^{\prime}+M_{2}$. Действительно, если $x_{0} \in F^{\prime}+M_{2}$, то конфигурация $x_{0}$ представима в виде $x_{0}=y_{0}+z_{0}$, где $y_{0} \in F^{\prime}, z_{0} \in M_{2}$. Так как $y_{0}$ - предельная точка в $F$, существует нетривиальная последовательность $\left\{y_{k}\right\}_{k=1}^{+\infty} \subset F, y_{k} \rightarrow y_{0}$ при $k \rightarrow \infty$. (Последовательность считается нетривиальной в том смысле, что ее предел является точкой накопления для элементов последовательности.) Тогда $\left(y_{k}+z_{0}\right) \in F+$ $M_{2}=E ; y_{k}+z_{0} \rightarrow y_{0}+z_{0}=x_{0}$ при $k \rightarrow \infty$, причем последовательность $\left\{y_{k}+z_{0}\right\}_{k=1}^{+\infty}$ нетривиальна. Таким образом, $x_{0}$-предельная точка в $E$, т.е. $x_{0} \in E^{\prime}$.

Для доказательства вложения $E^{\prime} \subset F^{\prime}+M_{2}$ рассмотрим произвольный элемент $x_{0}$ из $E^{\prime}$. Конфигурация $x_{0}$ - предельная точка для $E$, поэтому найдется нетривиальная (в том же смысле, что и вьше) последовательность $\left\{x_{k}\right\}_{k=1}^{+\infty} \subset E, x_{k} \rightarrow x_{0}$ при $k \rightarrow \infty$. Каждьй из $x_{k}$ представим в виде $x_{k}=y_{k}+z_{k}$, где $y_{k} \in F, z_{k} \in M_{2}$. Принимая во внимание компактность пространства $\Omega(G, A)$, получаем, что из $\left\{z_{k}\right\}_{k=1}^{+\infty}$ можно выделить сходящуюся подпоследовательность $\left\{z_{k_{j}}\right\}_{j=1}^{+\infty}$, пределом которой является некоторьй $z_{0} \in M_{2}$. Следовательно,

$$
x_{0}=\lim _{j \rightarrow \infty} x_{k_{j}}=\lim _{j \rightarrow \infty}\left(y_{k_{j}}+z_{k_{j}}\right)=\lim _{j \rightarrow \infty} y_{k_{j}}+z_{0}
$$


и существует

$$
\lim _{j \rightarrow \infty} y_{k_{j}}=y_{0}
$$

Возможны два случая: $z_{0} \neq \mathbf{0}$ и $z_{0}=\mathbf{0}$.

Если $z_{0} \neq \mathbf{0}$, то последовательность $\left\{z_{k_{j}}\right\}_{k=1}^{+\infty}$ с некоторого шага становится тождественной (существует $j_{0}$ такое, что при $j>j_{0}$ вьполнено $z_{k_{j}}=z_{0}$ ). В силу нетривиальности последовательности $\left\{x_{k}\right\}_{k=1}^{+\infty}$ конфигурации $y_{k_{j}} \neq y_{0}$ при $j>j_{0}$, поэтому $y_{0}$ является предельной точкой в $F$. Следовательно, $x_{0}=y_{0}+z_{0} \in F^{\prime}+M_{2}$.

Если $z_{0}=\mathbf{0}$, то $x_{0}=y_{0} \in F$. По предположению индукции $M_{\alpha-1} \in \widehat{\Sigma}$. При переходе к производному множеству свойства в) и г) из определения класса $\widehat{\Sigma}$ сохраняются, поэтому указанные свойства выполнены для всех множеств $M_{\alpha-1}^{(\beta)}$ при $\beta<\alpha-1$, а значит и для $F$. Пусть $\operatorname{supp}\left(x_{0}\right)=\left\{g_{1}, \ldots, g_{m}\right\}$. По свойству г) конфигурация $\widetilde{y}_{0}$, у которой $\operatorname{supp}\left(\widetilde{y}_{0}\right)=\left\{g_{1}, \ldots, g_{m-1}\right\}$, принадлежит $F^{\prime}$. Определим $\widetilde{z}_{0} \in M_{2}, \operatorname{supp}\left(\widetilde{z}_{0}\right)=\left\{g_{m}\right\}$. Получили, что $x_{0}=\widetilde{y}_{0}+\widetilde{z}_{0} \in F^{\prime}+M_{2}$.

Лемма доказана.

Лемма 4. Множество $M_{\alpha}$ лежит в классе $\Sigma$.

ДокАЗАтЕЛьство. Счетность и $T$-инвариантность множества $M_{\alpha}$ следуют из соответствующих свойств $M_{\alpha-1}$ и $M_{2}$. Докажем замкнутость построенного множества. Пусть $x_{k} \in M_{\alpha}, k=1,2, \ldots$, и $x_{k} \rightarrow x_{0}$ при $k \rightarrow \infty$. При каждом $k$ найдутся конфигурации $y_{k} \in M_{\alpha-1}$ и $z_{k} \in M_{2}$ такие, что $x_{k}=y_{k}+z_{k}$. В силу компактности множества $M_{2}$ существует подпоследовательность $z_{k_{j}}$, сходящаяся к некоторому $z_{0} \in M_{2}$. Так как $\left(y_{k_{j}}+z_{k_{j}}\right) \rightarrow x_{0}$ при $k \rightarrow \infty$, найдется $y_{0} \in M_{\alpha-1}$, к которому сходятся конфигурации $y_{k_{j}}$. Поэтому $x_{0}=y_{0}+z_{0} \in M_{\alpha-1}+M_{2}=M_{\alpha}$. Лемма доказана.

Лемма 5. Глубина множества $M_{\alpha}$ равна $\alpha$.

ДокАЗАТЕЛьСтво. Действительно,

$$
M_{\alpha}^{(\alpha-1)}=\left(\bigcap_{\beta<\alpha-1} M_{\alpha}^{(\beta)}\right)^{\prime}=\left(\bigcap_{\beta<\alpha-1} M_{\alpha-1}^{(\beta)}+M_{2}\right)^{\prime}=\bigcap_{\beta<\alpha-1} M_{\alpha-1}^{(\beta)}
$$

(второе равенство следует из леммы 3 , а третье-из того, что пересечение $\bigcap_{\beta<\alpha-1} M_{\alpha-1}^{(\beta)}$ конечно, поскольку его производное множество пусто). Значит, множество $M_{\alpha}^{(\alpha-1)}$ конечно, поэтому $M_{\alpha}^{(\alpha)}=\varnothing$. Следовательно, $\operatorname{depth}\left(M_{\alpha}\right)=\alpha$. Лемма доказана.

Лемма 6. Множество $M_{\alpha}$ лежит в классе $\widehat{\Sigma}$.

ДокАЗАТЕЛЬСтво. Счетность, замкнутость и $T$-инвариантность множества $M_{\alpha}$ доказаны в лемме 4 .

Докажем однородность глубины $M_{\alpha}$. Пусть $G_{0}$ - некоторое бесконечное подмножество групшы $G$. Так как по предположению индукции глубины множеств $M_{\alpha-1}$ и $M_{2}$ однородны, имеем $\operatorname{depth}\left(\left.M_{\alpha-1}\right|_{G_{0}}\right)=\alpha-1$ и $\operatorname{depth}\left(\left.M_{2}\right|_{G_{0}}\right)=2$. Из определения $M_{\alpha}$ следует, что $\left.M_{\alpha}\right|_{G_{0}}=\left.M_{\alpha-1}\right|_{G_{0}}+\left.M_{2}\right|_{G_{0}}$. Кроме того, множества $\left.M_{\alpha-1}\right|_{G_{0}}$ и $\left.M_{2}\right|_{G_{0}}$ счетны, замкнуты и обладают свойствами в) и г) из определения класса $\widehat{\Sigma}$. Рассуждая как и при доказательстве лемм $3-5$, получаем, что $\operatorname{depth}\left(\left.M_{\alpha}\right|_{G_{0}}\right)=\alpha$, т.е. множество $M_{\alpha}$ имеет однородную глубину. 
Конечность носителей конфигураций из $M_{\alpha}$ вытекает из того же свойства, выполненного по предположению индукции для конфигураций из $M_{\alpha-1}$ и из $M_{2}$.

Покажем, что множество $M_{\alpha}$ удовлетворяет свойству г) из определения класса $\widehat{\Sigma}$. Пусть $x \in M_{\alpha}$. Тогда либо $x \in M_{\alpha-1}$, либо найдутся конфигурации $y \in M_{\alpha-1} \mathrm{c}$ носителем $\operatorname{supp}(y)=\left\{g_{1}, \ldots, g_{n}\right\}$ и $z \in M_{2}$ с носителем $\operatorname{supp}(z)=\left\{g_{0}\right\}$ такие, что $\operatorname{supp}(x)=\operatorname{supp}(y) \cup \operatorname{supp}(z)$. В первом случае доказываемое свойство следует из вложения $M_{\alpha-1}^{\prime} \subset M_{\alpha}^{\prime}$ и того, что $M_{\alpha-1} \in \widehat{\Sigma}$. Во втором случае при $i=1, \ldots, n$ конфигурации с носителями $\left\{g_{1}, \ldots, g_{i-1}, g_{i+1}, \ldots, g_{n}, g_{0}\right\}$ являются предельными точками для $M_{\alpha}$, так как множество $M_{\alpha-1}$ удовлетворяет свойству г), и потому конфигурации с носителями $\left\{g_{1}, \ldots, g_{i-1}, g_{i+1}, \ldots, g_{n}\right\}$ суть предельные точки в нем.

Лемма доказана.

ЗАмЕЧАНИЕ. Из проведенных вьше рассуждений следует, что для произвольного натурального числа $n$ примером системы с однородной глубиной равной $n$ может служить система, фазовое пространство которой есть множество всех конфигураций, принимающих значение 1 не более чем на $n-1$ элементах группы $G$ :

$$
M_{n}:=\{x \in \Omega \mid \# \operatorname{supp}(x) \leqslant n-1\} \in \widetilde{\Sigma}(G, A), \quad \operatorname{depth}\left(M_{n}\right)=n .
$$

ii) Пусть $\alpha-1$ - предельный ординал. Введем в рассмотрение семейство отображений $R_{n}: \widehat{\Sigma}(G, A) \rightarrow \widehat{\Sigma}(G, A)$, занумерованных натуральными числами $n$ и действующих следуюшим образом:

$$
R_{n}(M)=\left\{x \in M \mid\left(g_{1}, g_{2} \in \operatorname{supp}(x), g_{1} \neq g_{2}\right) \Longrightarrow p\left(g_{1}, g_{2}\right)>n\right\} .
$$

Лемма 7. Определение отображсений $R_{n}$ корректно, т.е. все $R_{n}$ сохраняют свойства а)-г) из определения класса $\widehat{\Sigma}$. Кроме того, отображения $R_{n}$ не изменяют глубину любых множеств из $\widehat{\Sigma}$.

ДокАЗАТЕЛЬСТВо. Конечность либо счетность $R_{n}$-образа не более чем счетного множества $M$ из $\widetilde{\Sigma}(G, A)$ следует из очевидного вложения $R_{n} M \subset M$, вьполненного при любом $n$. Замкнутость и $T$-инвариантность $R_{n} M$ вытекают из соответствующих свойств $M$.

Покажем, что $R_{n} M$ обладает свойством однородности глубины. Пусть $n$-натуральное число и $G_{0}$ - некоторое бесконечное подмножество групшы $G$. Рассмотрим бесконечное множество $G_{n} \subset G_{0}$, в котором $p(g, h)>n$ для всех $g, h \in G_{n}$. Тогда $\left.\left(R_{n} M\right)\right|_{G_{n}}=$ $\left.M\right|_{G_{n}}$. Очевидно, что $\operatorname{depth}\left(\left.R_{n} M\right|_{G_{0}}\right) \leqslant \operatorname{depth}\left(R_{n} M\right)$. Вместес тем, $\operatorname{depth}\left(\left.R_{n} M\right|_{G_{0}}\right) \geqslant$ $\operatorname{depth}\left(\left.R_{n} M\right|_{G_{n}}\right)=\operatorname{depth}\left(\left.M\right|_{G_{n}}\right)=\operatorname{depth}(M) \geqslant \operatorname{depth}\left(R_{n} M\right)$ (предпоследнее равенство следует из однородности глубины $M)$. Тем самым, $\operatorname{depth}\left(\left.R_{n} M\right|_{G_{0}}\right)=\operatorname{depth}\left(R_{n} M\right)$.

Выполнение свойств в), г) из определения класса $\widehat{\Sigma}$ следует из построения отображений $R_{n}$.

Докажем инвариантность глубины множества из $\widehat{\Sigma}$ под действием $R_{n}$. Для каждого данного натурального $n$ рассмотрим бесконечное подмножество $G_{n}$ грушшы $G$, в котором для каждых $g, h \in G_{n}$ вьполнено $p(g, h)>n$. Так как глубина $M$ однородна, $\operatorname{depth}\left(\left.M\right|_{G_{n}}\right)=\operatorname{depth}(M)$. С другой стороны, элементы $\left.M\right|_{G_{n}}$ легко поднять до отображений из $G$ в $A$ при помощи $\eta: \Omega\left(G_{n}, A\right) \rightarrow \Omega(G, A)$,

$$
\eta(x)(g)= \begin{cases}x(g), & \text { если } g \in G_{n}, \\ 0 & \text { в противном случае. }\end{cases}
$$


Очевидно, при таком поднятии сохранится глубина: $\operatorname{depth}\left(\left.\eta M\right|_{G_{n}}\right)=\operatorname{depth}\left(\left.M\right|_{G_{n}}\right)$. Но $\left.\eta M\right|_{G_{n}} \subset R_{n} M$ по определению $R_{n}$ и по построению $G_{n}$, поэтому $\operatorname{depth}\left(\left.\eta M\right|_{G_{n}}\right) \leqslant$ $\operatorname{depth}\left(R_{n} M\right)$. Сравнивая это неравенство со следствием $\operatorname{depth}\left(R_{n} M\right) \leqslant \operatorname{depth}(M)$ вложения $R_{n} M \subset M$, приходим к выводу, что $\operatorname{depth}\left(R_{n} M\right)=\operatorname{depth}(M)$.

Лемма доказана.

Так как $\alpha$ - счетное трансфинитное число, все предшествуюшие ему непредельные ординалы можно занумеровать натуральными числами:

$$
\{\beta \mid \beta<\alpha, \beta \text { непредельньй }\}=\left\{\beta_{n}\right\}_{n=1}^{+\infty} .
$$

По предположению индукции для любого натурального $n$ существует $M_{\beta_{n}} \in \widehat{\Sigma}$ такое, что $\operatorname{depth}\left(M_{\beta_{n}}\right)=\beta_{n}$. Положим

$$
M_{\alpha}:=\bigcup_{n=1}^{+\infty} R_{n} M_{\beta_{n}}
$$

Лемма 8. Множество $M_{\alpha}$ находится в классе $\Sigma(G, A)$.

ДокАЗАТЕЛЬСтво. Очевидно, что $M_{\alpha}$ счетно и инвариантно относительно действия $T$.

Замкнутость $M_{\alpha}$ вытекает из следующих соображений. Допустим, что найдется последовательность $\left\{x_{k}\right\}_{k=1}^{+\infty} \subset M_{\alpha}$ такая, что ее пределом при $k \rightarrow+\infty$ является отображение $x_{0}$, не лежащее в $M_{\alpha}$. В $\left\{x_{k}\right\}_{k=1}^{+\infty}$ есть представители бесконечного набора множеств $L_{n}=R_{n} M_{\beta_{n}}$, так как в противном случае в одном из $L_{n}$ оказалась бы подпоследовательность $\left\{x_{k_{j}}\right\}_{j=1}^{+\infty}$, пределом которой при $j \rightarrow+\infty$ является $x_{0}$, что противоречит замкнутости рассматриваемого $L_{n}$ (которая имеет место в силу леммы 7 ).

Предельное соотношение $x_{k} \rightarrow x_{0}$ при $k \rightarrow+\infty$ означает, что для любого $N$ существует $K_{0}$ такое, что для всех $k>\left.K_{0} x_{k}\right|_{G_{N}}=\left.x_{K_{0}}\right|_{G_{N}}=\left.x_{0}\right|_{G_{N}}$, где $G_{N}=\{g \in G \mid$ $d(g) \leqslant N\}$ - шар радиуса $N$ в группе $G$.

Взяв $k>K_{0}$ такое, что $x_{k} \in L_{n}$ при некотором $n>2 N+2$, получим, что конфигурация $x_{0}$ может иметь на множестве $G_{N}$ не более одной единицы. В силу произвольности $N$ рассматриваемая $x_{0}$ есть либо тождественно нулевое отображение, либо обращается в 1 на единственном элементе групшы $G$. Но все такие конфигурации принадлежат построенному множеству $M_{2}$, поэтому $x_{0} \in M_{\alpha}$. Полученное противоречие доказьвает замкнутость $M_{\alpha}$.

Лемма доказана.

ЛЕмма 9. Глубина множества $M_{\alpha}$ равна $\alpha$.

ДокАЗАТЕЛЬСтво. Заметим, что полагая

$$
E:=\bigcap_{\beta<\alpha-1} M_{\alpha}^{(\beta)},
$$

получим $E=M_{2}$. В самом деле, предположим, что в $E$ содержится конфигурация $x_{0}$, обращающаяся в 1 не менее чем дважды (т.е. для некоторых $g_{1}, g_{2} \in G, g_{1} \neq g_{2}$, выполнено $\left.x_{0}\left(g_{1}\right)=x_{0}\left(g_{2}\right)=1\right)$. Конфигурация $x_{0}$ должна принадлежать бесконечному набору множеств $L_{n}$, так как в противном случае найдется шаг $\beta_{N}<\alpha-1$, на котором 
$x_{0}$ будет удалена из $M_{\alpha}^{\left(\beta_{N}\right)}$. Однако, $x_{0}$ не может принадлежать $L_{n}$ при $n>p\left(g_{1}, g_{2}\right)$. Получили противоречие.

$\mathrm{C}$ другой стороны, для всех ординалов $\beta$ меньших $\alpha-1$ имеет место вложение $M_{2} \subset$ $M_{\alpha}^{(\beta)}$ (поскольку при $n$ таких, что $\beta_{n}>\beta+1$, множество $M_{2}$ содержится в $L_{n}^{(\beta)}=$ $\left.\left(R_{n} M_{\beta_{n}}\right)^{(\beta)} \subset M_{\alpha}^{(\beta)}\right)$. Поэтому $E=M_{2}$.

Таким образом,

$$
\begin{gathered}
M_{\alpha}^{(\alpha-1)}=\left(\bigcap_{\beta<\alpha-1} M_{\alpha}^{(\beta)}\right)^{\prime}=M_{2}^{\prime}=M_{1}, \\
M_{\alpha}^{(\alpha)}=M_{\alpha}^{(\alpha-1)^{\prime}}=M_{1}^{\prime}=\varnothing, \quad \operatorname{depth}\left(M_{\alpha}\right)=\alpha .
\end{gathered}
$$

Лемма доказана.

Лемма 10. Множество $M_{\alpha}$ находится в классе $\widehat{\Sigma}(G, A)$.

ДокАЗАтЕльство. Счетность, замкнутость и $T$-инвариантность множества $M_{\alpha}$ доказаны в лемме 8. Выполнение свойств в), г) из определения класса $\widehat{\Sigma}$ следует из леммы 7 .

Докажем, что глубина $M_{\alpha}$ однородна. Пусть $G_{0}$ - бесконечное подмножество группы $G$. Заметим, что

$$
\left.M_{\alpha}\right|_{G_{0}}=\left.\bigcup_{n=1}^{+\infty}\left(R_{n} M_{\beta_{n}}\right)\right|_{G_{0}} .
$$

Рассуждая как и в лемме 9 , получаем (используя однородность глубин $M_{\beta_{n}}$ ), что

$$
\bigcap_{\beta<\alpha-1}\left(\left.M_{\alpha}\right|_{G_{0}}\right)^{(\beta)}=\left.M_{2}\right|_{G_{0}} .
$$

Следовательно, $\operatorname{depth}\left(\left.M_{\alpha}\right|_{G_{0}}\right)=\alpha=\operatorname{depth}\left(M_{\alpha}\right)$. Лемма доказана.

Таким образом, шаг трансфинитной индукции завершен. Истинность утверждения 1) теоремы установлена.

ЗАмЕчАнИЕ. Примером фазового пространства системы с однородной глубиной равной $\omega+1$ является

$$
\begin{aligned}
M_{\omega+1}:= & \{x \in \Omega \mid \# \operatorname{supp}(x)<\infty, \\
& \left.\operatorname{supp}(x)=\left\{g_{1}, \ldots, g_{n}\right\} \Longrightarrow p\left(g_{i}, g_{j}\right)>n+1,1 \leqslant i<j \leqslant n\right\} .
\end{aligned}
$$

2) Покажем, что не существует динамических систем со счетньм замкнутьм $T$-инвариантньм фазовым пространством, глубина которых была бы предельным трансфинитньм числом. Проведем рассуждения от противного. Допустим, что имеется множество $M$ со свойством $\operatorname{depth}(M)=\alpha$, где $\alpha$ - предельньй трансфинит, $M$ счетно, $T$-инвариантно и замкнуто. В таком случае множество $X:=\bigcap_{\beta<\alpha} M^{(\beta)}$ конечно $(X$ не может быть пустым как пересечение счетной системы убывающих компактов). Пусть $x_{0} \in X$. Тогда орбита точки $x_{0}$ под действием $T$ конечна. Следовательно, для каждого из образующих $c_{i} \in C$ группы $G$ найдется положительное число $P_{i}$ такое, что для всех $g \in G$ выполнено $x_{0}\left(g \cdot c_{i}^{P_{i}}\right)=x_{0}(g) ; P_{i}$ есть "период $x_{0}$ вдоль образующего $c_{i}$ ".

Определим несколько вспомогательных понятий, которьпи мы воспользуемся для завершения доказательства теоремы. 
ОПредЕлЕниЕ. Пусть задан набор $\varepsilon=\left(\varepsilon_{1}, \ldots, \varepsilon_{r}\right)$, состоящий из $\varepsilon_{i}= \pm 1$. Назовем ( полным) октантом, соответствующим набору $\varepsilon$, следующееподмножество групшы $G$ :

$$
\Delta(\varepsilon)=\left\{g=c_{1}^{i_{1}} \cdots c_{r}^{i_{r}} \mid i_{j}=\left\{\begin{array}{ll}
0,1,2, \ldots, & \text { если } \varepsilon_{j}=1, \\
0,-1,-2, \ldots, & \text { если } \varepsilon_{j}=-1,
\end{array} \quad j=1, \ldots, r\right\} .\right.
$$

ОПРЕДЕЛЕНИЕ. Пусть задан набор целых чисел $L=\left(l_{1}, \ldots, l_{r}\right)$. Назовем частичным октантом, соответствующим набору $L$, следующее подмножество групшы $G$ :

$$
\Delta_{p}(L)=\left\{g=c_{1}^{i_{1}} \cdots c_{r}^{i_{r}} \mid i_{j}=\left\{\begin{array}{l}
0,1,2, \ldots, l_{j}, \quad \text { если } l_{j}>0, \\
l_{j}, \ldots,-2,-1,0, \text { если } l_{j}<0,
\end{array} \quad j=1, \ldots, r\right\} .\right.
$$

ОПРЕДЕЛЕНИЕ. Назовем параллелепипедом размеров $l_{1}, \ldots, l_{r}$ (все $l_{j}$ - натуральные числа) следующее подмножество группы $G$ :

$$
\Pi_{l_{1}, \ldots, l_{r}}=\left\{g=c_{1}^{i_{1}} \cdots c_{r}^{i_{r}}|| i_{j} \mid \leqslant l_{j}, j=1, \ldots, r\right\} .
$$

Так как при всех $\beta<\alpha$ отображение $x_{0}$ является предельной точкой множеств $M^{(\beta)}$, то для любого $\beta<\alpha$ существует последовательность $\left\{x_{k}^{\beta}\right\}_{k=1}^{+\infty} \subset M^{(\beta)}$ такая, что $x_{k}^{\beta} \rightarrow$ $x_{0}$ при $k \rightarrow+\infty$, но $x_{k}^{\beta} \neq x_{0}$ для всех $k \in \mathbb{N}$.

Сходимость в рассматриваемом пространстве $\Omega$ является покоординатной, поэтому (при необходимости переходя к подпоследовательности) можно считать, что $x_{k}^{\beta}$ совпадают с $x_{0}$ на "кубах размера $k$ " : для любого $\beta<\alpha$

$$
\left.x_{k}^{\beta}\right|_{\Pi_{k, \ldots, k}}=\left.x_{0}\right|_{\Pi_{k, \ldots, k}} .
$$

Поясним теперь схему дальнейших рассуждений. Будем сдвигать конфигурации $x_{k}^{\beta}$ вдоль каждой из образующих в ту или иную сторону (в положительном или отрицательном направлении) на максимально возможное количество периодов с тем, чтобы получить конфигурации, совпадающие с $x_{0}$ на достаточно больших частичных октантах, но имеющие отличие от $x_{0}$ в параллелепипеде $\Pi_{P_{1}, \ldots, P_{r}}$ (такие сдвиги $x_{k}^{\beta}$ всегда найдутся, потому что $x_{k}^{\beta} \neq x_{0}$ ). При увеличении $k$ размеры частичных октантов, на которых есть совпадение сдвинутых $x_{k}^{\beta}$ с $x_{0}$, будут расти. В силу замкнутости и $T$-инвариантности множества $M^{(\beta)}$ найдутся конфигурации $y^{\beta} \in M^{(\beta)}$, совпадающие с $x_{0}$ в одном из полных октантов, но имеющие отличие от него в $\Pi_{P_{1}, \ldots, P_{r}}$. Можно выбрать октант $\Delta\left(\varepsilon_{0}\right)$ такой, что при всех $\beta<\alpha$ в $M^{(\beta)}$ есть конфигурация $y^{\beta}$, совпадающая в нем с $x_{0}$. Тогда и в $X=\bigcap_{\beta<\alpha} M^{(\beta)}$ существует конфигурация $y_{0} \in X$ такая, что $\left.y_{0}\right|_{\Delta\left(\varepsilon_{0}\right)}=\left.x_{0}\right|_{\Delta\left(\varepsilon_{0}\right)}$, при этом найдется $g \in \Pi_{P_{1}, \ldots, P_{r}}$, на котором $y_{0}(g) \neq x_{0}(g)$. Орбита $y_{0}$ под действием $T$ бесконечна, что противоречит конечности $T$-инвариантного множества $X$.

Проведем более детальное обоснование обозначенных результатов.

ЛЕмма 11. При всех $k>\max \left\{P_{1}, \ldots, P_{r}\right\}$ для каждого $x_{k}^{\beta}$ найдутся әлемент $g^{*} \in G$ и набор иелых чисел $S=S(k)=\left\{s_{1}(k), \ldots, s_{r}(k)\right\}$ такие, что для конфигурачии $y_{k}^{\beta}=T_{g^{*}} x_{k}^{\beta}$ выполнены следуюшие условия:

a) $\left.y_{k}^{\beta}\right|_{\Delta_{p}(S)}=\left.x_{0}\right|_{\Delta_{p}(S)}$;

б) существует $g_{0} \in \Pi_{P_{1}, \ldots, P_{r}}$ maкое, что $y_{k}^{\beta}\left(g_{0}\right) \neq x_{0}\left(g_{0}\right)$;

в) для всех $j=1, \ldots, r\left|s_{j}(k)\right| \rightarrow \infty$ nрu $k \rightarrow \infty$. 
ДокАЗАТЕЛьСтво. Выберем натуральные числа $m_{1}, \ldots, m_{r}$ так, чтобы в параллелепипеде $\Pi_{m_{1} P_{1}}, \ldots, m_{r} P_{r}$ имело место совпадение конфигураций $x_{0}$ и $x_{k}^{\beta}$, а в параллелепипеде $\Pi_{\left(m_{1}+1\right) P_{1}, \ldots,\left(m_{r}+1\right) P_{r}}$ был элемент $\widetilde{g}=c_{1}^{a_{1} P_{1}+b_{1}} \cdots c_{r}^{a_{r} P_{r}+b_{r}}\left(\left|a_{j}\right| \leqslant m_{j}\right.$, $\left|b_{j}\right| \leqslant P_{j}$ при $\left.j=1, \ldots, r\right)$, на котором $x_{0}(\widetilde{g}) \neq x_{k}^{\beta}(\widetilde{g})$. Обозначим для краткости $\Pi_{(1)}:=$ $\Pi_{m_{1} P_{1}, \ldots, m_{r} P_{r}}, \Pi_{(2)}:=\Pi_{\left(m_{1}+1\right) P_{1}, \ldots,\left(m_{r}+1\right) P_{r}}, \Pi_{(k)}:=\Pi_{k, \ldots, k}$.

Так как по построению $x_{0}$ и $x_{k}^{\beta}$ совпадают на $\Pi_{(k)}$, имеем $\Pi_{(k)} \subset \Pi_{(2)}$, откуда получаем неравенства

$$
k<\left(m_{j}+1\right) P_{j}, \quad m_{j}>\frac{k}{P_{j}}-1, \quad j=1, \ldots, r .
$$

Положим

$$
g^{*}:=c_{1}^{-a_{1} P_{1}} \cdots c_{r}^{-a_{r} P_{r}}, \quad s_{j}:=\left\{\begin{array}{l}
P_{j}-k, \text { если } a_{j} \geqslant 0 \\
k-P_{j}, \text { если } a_{j}<0
\end{array}\right.
$$

Подействуем на равенство $\left.x_{0}\right|_{\Pi_{(1)}}=\left.x_{k}^{\beta}\right|_{\Pi_{(1)}}$ преобразованием $T_{g^{*}}$ :

$$
\left.\left(T_{g^{*}} x_{0}\right)\right|_{g^{*} \Pi_{(1)}}=\left.\left(T_{g^{*}} x_{k}^{\beta}\right)\right|_{g^{*} \Pi_{(1)}} .
$$

Конфигурация $x_{0}$ инвариантна относительно сдвигов вдоль любого из образуюших на произвольное количество соответствующих периодов, поэтому $T_{g^{*}} x_{0}=x_{0}$. Учитывая, что по определению $y_{k}^{\beta}=T_{g^{*}} x_{k}^{\beta}$, получаем, что

$$
\left.x_{0}\right|_{g * \Pi_{(1)}}=\left.y_{k}^{\beta}\right|_{g^{*} \Pi_{(1)}} .
$$

а) Докажем, что $\Delta_{p}(S) \subset g^{*} \Pi_{(1)}$. Тем самьм, будет установлено условие а) леммы 11.

Пусть $g \in \Delta_{p}(S)$. Представим $g$ в виде $g=c_{1}^{i_{1}} \cdots c_{r}^{i_{r}}$, где

$$
i_{j} \in\left\{\begin{array}{l}
\left\{0, \ldots, s_{j}\right\}, \text { если } s_{j} \geqslant 0, \\
\left\{s_{j}, \ldots, 0\right\}, \text { если } s_{j}<0,
\end{array} \quad j=1, \ldots, r,\right.
$$

т.е.

$$
i_{j} \in\left\{\begin{array}{l}
\left\{0, \ldots, k-P_{j}\right\}, \text { если } a_{j}<0, \\
\left\{P_{j}-k, \ldots, 0\right\}, \text { если } a_{j} \geqslant 0,
\end{array} \quad j=1, \ldots, r .\right.
$$

Элементы же из $g^{*} \Pi_{(1)}$ имеют вид $c_{1}^{q_{1}} \cdots c_{r}^{q_{r}}, q_{j} \in\left\{-m_{j} P_{j}-a_{j} P_{j}, \ldots, m_{j} P_{j}-a_{j} P_{j}\right\}$, $j=1, \ldots, r$. Зафиксируем некоторое $j, 1 \leqslant j \leqslant r$. Покажем, что в проекции на $j$-й образуюший элемент доказываемое вложение вьполнено. Возможны два случая: $a_{j}<0$ и $a_{j} \geqslant 0$.

В случае $a_{j}<0$ докажем, что $\left\{0, \ldots, k-P_{j}\right\} \subset\left\{-m_{j} P_{j}-a_{j} P_{j}, \ldots, m_{j} P_{j}-a_{j} P_{j}\right\}$. Так как по построению $\left|a_{j}\right| \leqslant m_{j}$, имеем $0 \leqslant-a_{j} \leqslant m_{j}$. Поэтому

$$
-m_{j} P_{j}-a_{j} P_{j} \leqslant-m_{j} P_{j}+m_{j} P_{j}=0, \quad m_{j} P_{j}-a_{j} P_{j} \geqslant m_{j} P_{j}>\left(\frac{k}{P_{j}}-1\right) P_{j}=k-P_{j} .
$$


В случае $a_{j} \geqslant 0$ докажем, что $\left\{P_{j}-k, \ldots, 0\right\} \subset\left\{-m_{j} P_{j}-a_{j} P_{j}, \ldots, m_{j} P_{j}-a_{j} P_{j}\right\}$. Заметим, что $0 \leqslant a_{j} \leqslant m_{j}$. Имеем

$-m_{j} P_{j}-a_{j} P_{j} \leqslant-m_{j} P_{j}<-\left(\frac{k}{P_{j}}-1\right) P_{j}=P_{j}-k, \quad m_{j} P_{j}-a_{j} P_{j} \geqslant m_{j} P_{j}-m_{j} P_{j}=0$.

В силу произвольности $j$ доказываемое вложение установлено.

б) Положим $g_{0}:=g^{*} \widetilde{g}$. Так как по построению $x_{0}(\widetilde{g}) \neq x_{k}^{\beta}(\widetilde{g})$, имеем

$$
x_{0}\left(g_{0}\right)=x_{0}\left(g^{*} \widetilde{g}\right)=x_{0}(\widetilde{g}) \neq x_{k}^{\beta}(\widetilde{g})=\left(T_{g^{*}} x_{k}^{\beta}\right)\left(g^{*} \widetilde{g}\right)=y_{k}^{\beta}\left(g_{0}\right) .
$$

При этом

$$
g_{0}=\left(c_{1}^{a_{1} P_{1}+b_{1}} \cdots c_{r}^{a_{r} P_{r}+b_{r}}\right) \cdot\left(c_{1}^{-a_{1} P_{1}} \cdots c_{r}^{-a_{r} P_{r}}\right)=c_{1}^{b_{1}} \cdots c_{r}^{b_{r}} .
$$

По построению $\left|b_{j}\right| \leqslant P_{j}, j=1, \ldots, r$. Поэтому $g_{0} \in \Pi_{P_{1}, \ldots, P_{r}}$. Условие б) установлено.

в) Условие в) следует из явного вида чисел $s_{j}$, так как $\left|s_{j}(k)\right|=k-P_{j} \rightarrow \infty$ при $k \rightarrow \infty$.

Лемма доказана.

Рассмотрим при каждом $\beta<\alpha$ множество

$$
E^{\beta}=\left\{\varepsilon \in\{ \pm 1\}^{r}\left|\exists y^{\beta} \in M^{(\beta)}: y^{\beta}\right|_{\Delta(\varepsilon)}=\left.x_{0}\right|_{\Delta(\varepsilon)},\left.y^{\beta}\right|_{\Pi_{P_{1}, \ldots, P_{r}}} \neq\left. x_{0}\right|_{\Pi_{P_{1}, \ldots, P_{r}}}\right\} .
$$

Лемма 12. Множества $E^{\beta}$ обладают следующими свойствами:

а) для любого ординала $\beta<\alpha$ множество $E^{\beta}$ непусто;

б) если $\beta_{1}<\beta_{2}, m o E^{\beta_{1}} \supset E^{\beta_{2}}$.

ДокАЗАТЕЛЬСтво. а) Зафиксируем некоторое $\beta<\alpha$. При каждом $k=1,2, \ldots$ найдется октант, в частичном подоктанте которого имеется совпадение $y_{k}^{\beta}$ и $x_{0}$ (сохраним обозначения, введенные в лемме 11). Так как имеется лишь конечное число октантов, при некотором $\varepsilon=\varepsilon_{0}$ для бесконечного набора индексов $k=k_{j}, j=1,2, \ldots$, частичные октанты $\Delta_{p}\left(S\left(k_{j}\right)\right)$ лежат внутри $\Delta\left(\varepsilon_{0}\right)$. В силу того что пространство $M^{(\beta)}$ компактно, у последовательности $\left\{y_{k_{j}}^{\beta}\right\}_{j=1}^{+\infty}$ есть предельная точка $y^{\beta}$, совпадающая с конфигурацией $x_{0}$ на октанте $\Delta\left(\varepsilon_{0}\right)$, но имеющая отличие от $x_{0}$ в $\Pi_{P_{1}, \ldots, P_{r}}$. В силу замкнутости множества $M^{(\beta)}$ конфигурация $y^{\beta} \in M^{(\beta)}$.

б) Указанное вложение легко следует из того, что $M^{\left(\beta_{1}\right)} \supset M^{\left(\beta_{2}\right)}$ при $\beta_{1}<\beta_{2}$.

Лемма доказана.

Введем в рассмотрение следующие множества не более чем счетных ординалов, зависящие от наборов $\varepsilon \in\{ \pm 1\}^{r}$ :

$$
K^{\varepsilon}=\left\{\beta<\alpha\left|\exists y^{\beta} \in M^{(\beta)}: y^{\beta}\right|_{\Delta(\varepsilon)}=\left.x_{0}\right|_{\Delta(\varepsilon)},\left.y^{\beta}\right|_{\Pi_{P_{1}, \ldots, P_{r}}} \neq\left. x_{0}\right|_{\Pi_{P_{1}, \ldots, P_{r}}}\right\} .
$$

Лемма 13. Найдется набор $\varepsilon_{0}$ из \pm 1 такой, что $K^{\varepsilon_{0}}=\{\beta \mid \beta<\alpha\}$.

ДоКАЗАТЕЛЬСТво. Из утверждений а) и б) леммы 12 следует соответственно, что $\bigcup_{\varepsilon} K^{\varepsilon}=\{\beta \mid \beta<\alpha\}$ и для любого $\varepsilon$ существует $\beta_{0}$ такое, что $K^{\varepsilon}=\left\{\beta \mid \beta<\beta_{0}\right\}$. Сопоставляя эти два факта, получаем утверждение леммы. Лемма доказана. 
СлЕДСТВИЕ. Существует октант $\Delta\left(\varepsilon_{0}\right)$ такой, что при всех $\beta<\alpha$ в множестве $M^{(\beta)}$ имеется конфигурация $y^{\beta}$, совпадающая с $x_{0}$ в $\Delta\left(\varepsilon_{0}\right)$, но имеющая отличие от него в $\Pi_{P_{1}, \ldots, P_{r}}$.

Нам потребуется также следующее утверждение из общей теории топологических пространств.

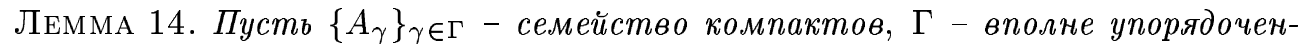
ное мнохество, точная нижняя грань которого есть $\gamma_{0}$, а все мнохества $A_{\gamma}$ непусты и убывают: $A_{\gamma_{1}} \supset A_{\gamma_{2}}$, если $\gamma_{1}<\gamma_{2}$. Тогда

$$
\bigcap_{\gamma \in \Gamma} A_{\gamma} \neq \varnothing
$$

ДокАЗАтЕЛЬСтво. Действительно, если предположить противное, то множества $A_{\gamma_{0}} \backslash A_{\gamma}$ образуют открытое покрытие компакта $A_{\gamma_{0}}$ в естественной топологии на $A_{\gamma_{0}}$. Выбирая конечное подпокрытие, приходим в противоречие с теоремой Кантора о пересечении счетной системы убывающих компактов. Лемма доказана.

ЛЕмма 15. Найдется конфигураиия $y_{0} \in X$ такая, что $\left.y_{0}\right|_{\Delta\left(\varepsilon_{0}\right)}=\left.x_{0}\right|_{\Delta\left(\varepsilon_{0}\right)}$, но $\left.y_{0}\right|_{\Pi_{P_{1}, \ldots, P_{r}}} \neq\left. x_{0}\right|_{\Pi_{P_{1}, \ldots, P_{r}}}$.

ДокАЗАтЕЛьСтво. Рассмотрим множества

$$
Y^{\beta}=\left\{y^{\beta} \in M^{(\beta)}\left|y^{\beta}\right|_{\Delta\left(\varepsilon_{0}\right)}=\left.x_{0}\right|_{\Delta\left(\varepsilon_{0}\right)},\left.y^{\beta}\right|_{\Pi_{P_{1}, \ldots, P_{r}}} \neq\left. x_{0}\right|_{\Pi_{P_{1}, \ldots, P_{r}}}\right\}
$$

В силу следствия из леммы 13 все $Y^{\beta} \neq \varnothing$. Так как множества $M^{(\beta)}$ убьвают, $Y^{\beta}$ тоже убьвают. Кроме того, очевидно, что множества $Y^{\beta}$ замкнуты. По лемме 14 их пересечение непусто. Лемма доказана.

Орбита под действием $T$ полученной конфигурации $y_{0}$ бесконечна. Однако, в силу $T$-инвариантности множества $X$ вся эта орбита должна лежать в нем. Полученное противоречие с конечностью $X$ полностью доказывает теорему.

Пользуясь случаем, автор анонсирует следующий результат. Применив конструкцию, аналогичную использованной в настоящей работе, автор получил новое решение задачи Биркгофа о множестве значений глубины центра, опирающееся на методы символической динамики. На наш взгляд, получающаяся при этом конструкция существенно проще построения Майера. Статья, в которой изложен этот результат, в данньй момент готовится к печати.

В заключение автор благодарит Д. В. Аносова и А. М. Стёпина, привлекших его внимание к изучению инварианта глубины, а также Г. Чакветадзе, ознакомившегося с рукописью и сделавшего ряд ценных замечаний. 


\section{СПИСОК ЦИТИРОВАННОЙ ЛИТЕРАТУРЫ}

[1] Шаповалов С. А. Сопряженность символических динамических систем не более чем со счетным фазовым пространством // Вестн. МГУ. Сер. 1. Матем. , мех. 1997. №3. С. 35-39.

[2] Birkhoff G.D. Über gewisse Zentralbewegungen Dynamischer Systeme // Ges. Wiss. Göttingen Nachr. Math. -Phys. Kl. 1926. P. 81-92.

[3] Майер А. Г. О порядковом числе центральных траекторий // Докл. АН СССР. 1948. Т. 59. № 8. C. $1393-1396$.

[4] Немыцкий В. В., Степанов В.В. Качественная теория дифференциальных уравнений. 2-е изд. М.-Л.: ГИТТЛ, 1949.

[5] Neumann D. A. Central sequences in dynamical systems // Amer. J. Math. 1978. V. 100. № 1. P. 1-18.

[6] Anosov D. V. Remarks concerning hyperbolic sets // J. Math. Sci. 1996. V. 78. №5. P. 497-529. 